\title{
INVESTIGACIÓN EN JÍCAMA (Pachyrhizus erosus) Y SU POTENCIAL PARA EL DESARROLLO AGRÍCOLA DE LA REGIÓN1
}

\author{
Antonio Mora ${ }^{2}$, Jorge Morera ${ }^{2}$
}

\begin{abstract}
RESUMEN
Investigación en jícama (Pachyrhizus erosus) y su potencial para el desarrollo agrícola de la Región. La Jícama (Pachyrhizus erosus) representa una excelente alternativa para la diversificación de la agricultura en el trópico americano, ya que es posible utilizar sus raíces tuberosas como alimento, y de las semillas obtener rotenona para la elaboración de productos insecticidas naturales. El presente trabajo tiene como objeto informar los últimos adelantos de investigación obtenidos en la evaluación de nuevos genotipos de jícama bajo diferentes condiciones ambientales de Costa Rica. Durante abril y julio de 1993 se establecieron tres experimentos: los primeros dos consideraron la evaluación de 10 genotipos bajo dos localidades, Alajuela y Turrialba. El tercer experimento en Turrialba consideró distancias de siembra $(0,10,0,15$ y $0,20 \mathrm{~m}$ entre plantas). En general, existió una tendencia muy marcada respecto a la localidad; en ensayos consecutivos se ha comprobado que la zona de Alajuela es la más apropiada para el desarrollo del cultivo de la jícama. Referente a las distancias de siembra los resultados preliminares sugieren que a menor distancia de siembra mayor es la producción de raíces, y menor el peso de las mismas. Para el desarrollo agrícola regional la Jícama ofrece una alternativa de tomar en cuenta, ya que es una especie que se adapta bien dentro de los sistemas de los agricultores, mejorando la sostenibilidad alimenticia.
\end{abstract}

\begin{abstract}
Research with yam bean (Pachyrhizus erosus) and its potential for the agricultural growth of the agricultural growth of the region. The yam bean (Pachyrhizus erosus) is an excellent alternative crop for the agricultural diversification in the American tropics, because the tuberous roots can be used as food and natural insecticides are produced with the rotenone extracted from the seeds. The main object of this trial was to submit the latest research findings obtained from the testing of new yam bean genotypes, under different environments in Costa Rica. Three experiments were established during April and July of 1993: 10 genotypes were evaluated at two localities, Alajuela and Turrialba, in the first two trials. The third trial conducted in Turrialba consisted of planting distances $(0.10,0.15$ and $0.20 \mathrm{~m}$ between plants. As a whole, there is a marked tendency with respect to the locality, as it was proven in consecutive trials that Alajuela is the most appropiated zone for growing yam bean. With reference to planting distances, the preliminary results suggest that at shorter distances, the larger the root production and the lower their weight. The yam bean offers an alternative to be considered for the agricultural development of the region because of itsadaptation within the farmers', systems, improving the food sustainability.
\end{abstract}

\section{INTRODUCCION}

Desde 1989 se han realizado varios ensayos con jícama, en Costa Rica donde se han encontrado amplias diferencias en rendimiento entre las diferentes introducciones evaluadas. Las primeras investigaciones mostraron bajos rendimientos generales, ocasionado principalmente por el uso de bajas densidades de siembra; sin embargo, a medida que estas densidades fueron aumentándose incremen- taron los rendimientos hasta obtener altas producciones (Morera 1992, Mora y Morera 1994).

Los espaciamientos recomendados son muy variables y éstos dependen de la longitud del periodo de crecimiento, el tamaño deseado de las raíces y la duración del día en el momento de la siembra (Heredia 1985, Sahadevan 1987).

\footnotetext{
1 Presentado en la XL Reunión Anual del PCCMCA en Costa Rica, América Central. 13 al 19 de marzo, 1994.

2 CATIE, Unidad Recursos Fitogenéticos.
} 
Arguelles (1979), Díaz (1978) y Heredia (1985) en México, recomiendan para la obtención de jícamas grandes, sembrar a doble hilera sobre surcos distanciados 92 $\mathrm{cm}$, con una distancia entre hileras de $25 \mathrm{~cm}$ y depositando una semilla cada $20 \mathrm{~cm}$. Para obtener jícamas pequeñas o "piñatera", recomiendan la siembra a doble hilera, sobre surcos distanciados $76 \mathrm{~cm}$ y a una distancia entre hileras de 20 a $25 \mathrm{~cm}$ y depositando una semilla cada 15 $\mathrm{cm}$.

En la India las distancias usualmente empleadas son de 30 a $40 \mathrm{~cm}$ entre plantas y de 60 a $75 \mathrm{~cm}$ entre hileras. En Filipinas es común el espaciamiento de $10 \mathrm{~cm}$ entre plantas y de 15 a $20 \mathrm{~cm}$ entre hileras, aunque en este mismo país, se ha demostrado experimentalmente, que la producción de raíces se duplica usando un espaciamiento de 15 x 15 cm (Kay 1973).

Debe existir un límite máximo de densidad, al igual que en otros cultivos de raíces y tubérculos, arriba de la cual los rendimientos pueden ser altos pero donde los tamaños y la calidad del producto es inferior. De aquí la necesidad de evaluar densidades o distancias de siembra para observar bajo cual condición se logran óptimas cosechas sin detrimento de la calidad.

El objetivo de ambos estudios fue probar el comportamiento agronómico de algunas introducciones bajo diferentes condiciones ambientales y en casos específicos delucidar el comportamiento a distintas densidades de siembra.

\section{MATERIALES Y MÉTODOS}

\section{Comportamiento agronómico de diez introducciones de jícama bajo dos localidades de Costa Rica.}

Se estableció un experimento para evaluar diez nuevas introducciones o líneas en dos localidades (EC-557; EC-236; EC-550; EC-533; EC-204; EC-214; EC-120-A; EC-503 y EC-117): Alajuela (Estación Experimental Fabio Baudrit M, UCR) y Turrialba (Cabiria, CATIE). Para cada ensayo se utilizó un diseño de bloques al azar con cuatro repeticiones.

En Alajuela la distancia entre surcos fue de $0,80 \mathrm{~m}$ y en Turrialba de 0,75 m Con las plantas espaciadas a 0,20 m entre sí; en cada surco se sembró doble hilera de plantas.

La siembra se realizó en Turrialba el 27 de abril y se cosechó el18 de octubre de 1993, en Alajuela la siembra fue el 13 de julio y la cosecha el 21 de diciembre del mismo año.

\section{Comportamiento agronómico de tres introducciones de jícama bajo tres distancias de siembra.}

El experimento se estableció en Cabiria, CATIE el 6 de junio y se cosechó el 24 de noviembre de 1993.

La prueba consistió en evaluar el efecto de tres distancias de siembra entre plantas (densidades) y tres introducciones. Se utilizó un experimento factorial 32 en un diseño de parcelas divididas con tres repeticiones. Los factores evaluados fueron: introducciones (tres niveles) en las parcelas grandes o completas y distancias (tres niveles) en las parcelas menores o subunidades. Se emplearon las introducciones EC-032, EC-509 y EC-534 con las distancias entre plantas de $0,10 \mathrm{~m}, 0,15 \mathrm{~m}$ y $0,20 \mathrm{~m}$; la distancia entre surcos fue de $0,75 \mathrm{~m}$.

Es importante aclarar que en cada surco se sembró doble hilera de plantas con una separación entre estas hileras de 0,25 m. Esto origina una densidad de siembra de 266667 plantas/ha con la distancia de 0,10 m, 177778 plantas/ha con $0,15 \mathrm{~m}$ y 133333 plantas/ha con la distancia $0,20 \mathrm{~m}$.

Durante la cosecha se clasificaron las raíces tuberosas en dos grupos de acuerdo al peso y tamaño: tamaño pequeño (200 g) y tamaño mediano (200-1000 g).

Las variables evaluadas fueron: número de raíces de tamaño pequeño, mediano y total; peso de raíces pequeñas, medianas y total. 


\section{RESULTADOS Y DISCUSION}

\section{Comportamiento agronómico de diez introducciones de jícama bajo condiciones ambientales de Costa Rica.}

El análisis de varianza para la variable peso de raíces $(\mathrm{kg} / \mathrm{ha})$ indicó diferencias significativas entre las introducciones en Turrialba y altamente significativas en Alajuela.

En la Figura 1 se comparan los rendimientos para diez tratamientos en ambas localidades. En Alajuela la producción de raíces tuberosas alcanzó los $60000 \mathrm{~kg} / \mathrm{ha}$ con la introducción EC-557; el menor rendimiento fue de $31239 \mathrm{~kg} / \mathrm{ha}$ con la introducción EC-117. En Turrialba el mejor promedio fue de $38334 \mathrm{~kg} / \mathrm{ha}$ de EC-550 y estadísticamente diferente del resto de promedios; los cuales entre sí, no mostraron diferencia estadística (Duncan, $\mathrm{p} \leq 0,05)$.

Al igual que en ensayos anteriores, la localidad de Alajuela presenta condiciones muy favorables para obtener altos rendimientos, el promedio general fue de 48835 $\mathrm{kg} / \mathrm{ha}$, aproximadamente el doble de lo obtenido en Turrialba. Asimismo, algunas introducciones presentaron producciones superiores al 50\% con respecto a Turrialba (Figura 1).

Adicionalmente, se observa que las introducciones variaron en rendimiento según la zona; así por ejemplo, las líneas EC-557 y EC-236 ocuparon los mejores rendimientos en Alajuela, pero, estuvieron en la cuarta y sexta posición respectivamente en Turrialba. El más bajo rendimiento de EC-117 en Alajuela fue el quinto en Turrialba.

Para futuros experimentos será necesario comparar localidades durante el mismo período (igual fecha de siembra y cosecha), así como evaluar épocas de siembra dentro de cada localidad.

\section{Comportamiento agronómico de tres introducciones de jícama bajo diferentes densidades de siembra.}

De acuerdo al análisis de varianza se presentaron diferencias altamente significativas $(1 \%)$ entre distancias para las variables número y peso de raíces de tamaño pe-

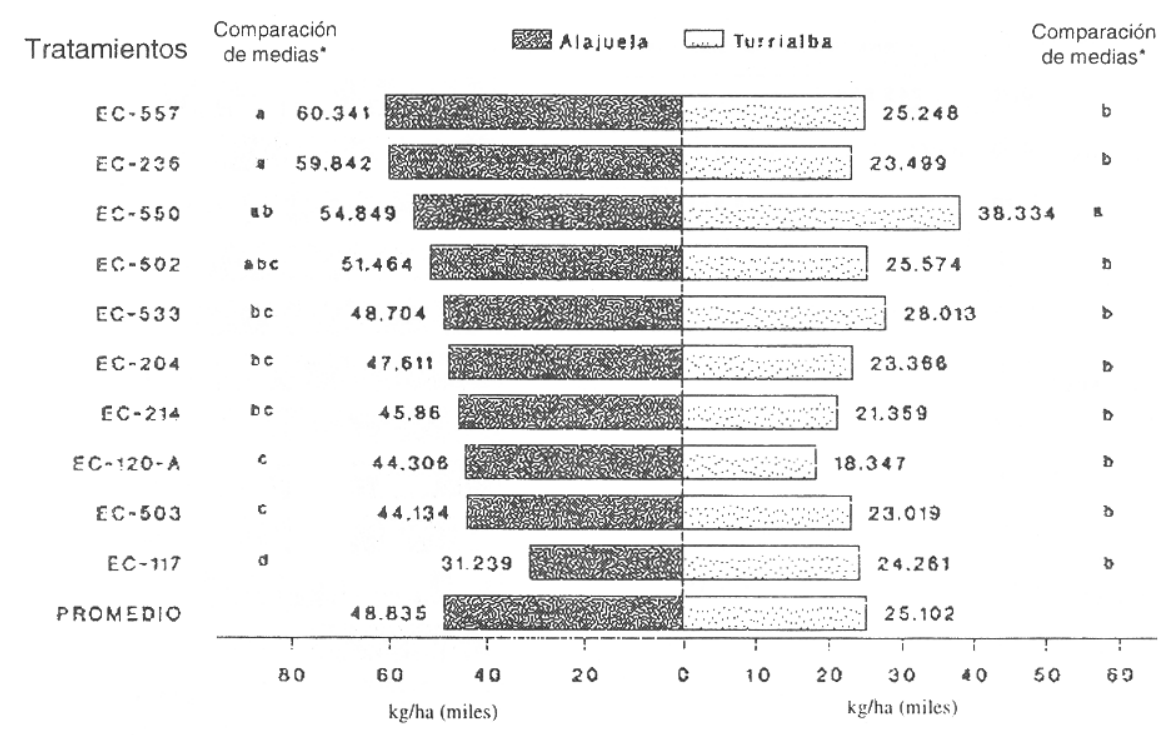

Fig. 1. Rendimiento promedio en peso fresco de raíces tuberosas de diez introducciones de jícama evaluadas en Alajuela y Turrialba. Unidad de Recursos Fitogenéticos, CATIE. 1993.

* Medias con la misma letra se consideran estadísticamente iguales 
queño y para el número de raíces totales. Diferencia significativa (5\%) se obtuvo entre distancias para el peso total de raíces e igualmente entre introducciones para el número total de raíces. Además, se obtuvo efecto significativo para la interacción de introducciones por distancias en las variables número de raíces de tamaño pequeño y para el total de raíces.

En los totales de número de raíces se encontró que las introducciones EC-032 y EC-509 tienen mayor cantidad de raíces que EC-534 superando a esta última por 38389 Y 30577 raíces/ha respectivamente (Figura 2).

Los promedios por introducción para el peso total fueron muy similares con el valor más bajo de 27955 kg/ha de EC-534; EC-509 mostró un peso de 31851 kg/ha ligeramente superior que EC-032 (31562 kg/ha) aunque esta última introducción fue superior en número de raíces, lo cual indica que el peso por raíz es más alto en EC-509.
De estos resultados se puede inferir que introducciones con el mismo rendimiento en peso pueden ser muy diferentes en el tamaño de raíces lo cual es muy significativo al momento de seleccionar un determinado genotipo.

$\mathrm{Al}$ igual que se mostró para el tamaño pequeño la tendencia para los totales es una disminución en el número de raíces en la medida que aumenta la distancia de siembra (Figura 2).

En la Figura 3 se comparan los promedios obtenidos para los tamaños y totales con cada distancia de siembra. El peso y número de raíces de tamaño pequeño fue alto en la medida que la distancia de siembra fue menor, lo cual significa que a . menor distancia mayor competencia entre plantas y por lo tanto más cantidad de raíces pequeñas. Para el tamaño mediano no se presenta diferencia significativa pero existe la tendencia contraria, o sea un número y peso de raíces inferior en la distancia más baja. Se puede inferir, igual al caso anterior, que las raíces tienden a

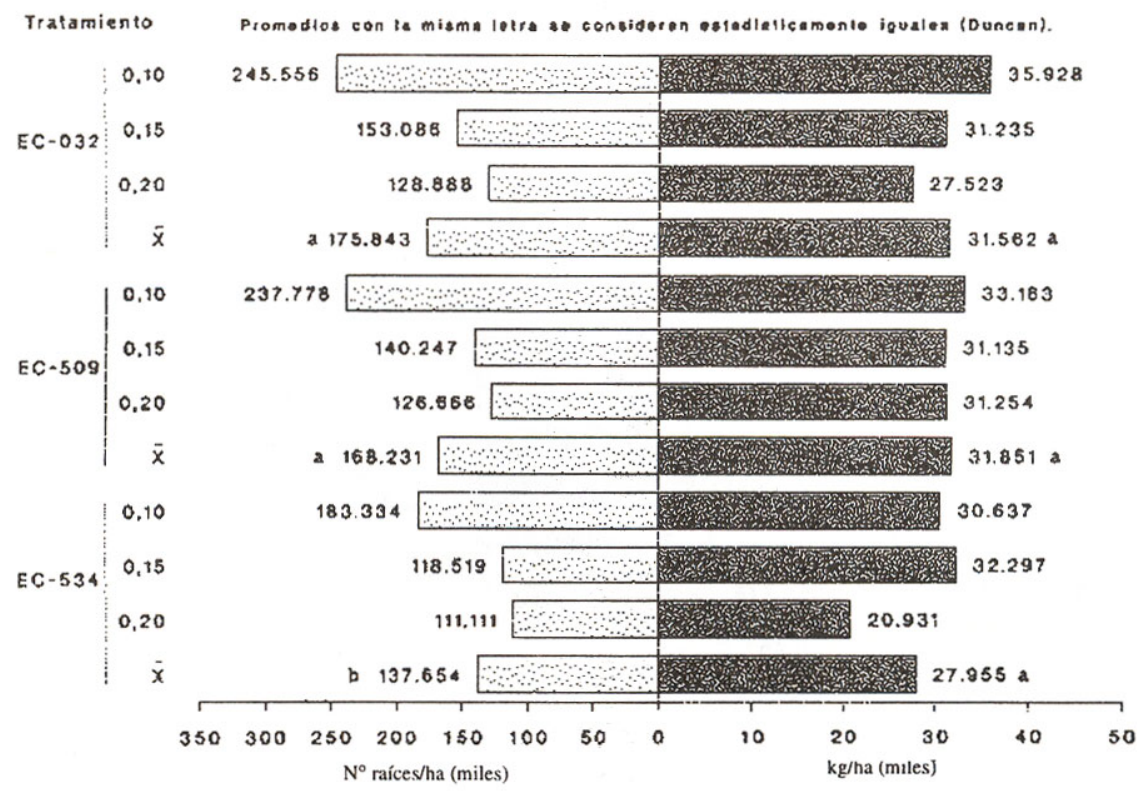

Fig. 2. Rendimientos promedio del número y peso de raíces totales para tres introducciones de jícama con tres distancias de siembra entre plantas. CATIE. 1993. 


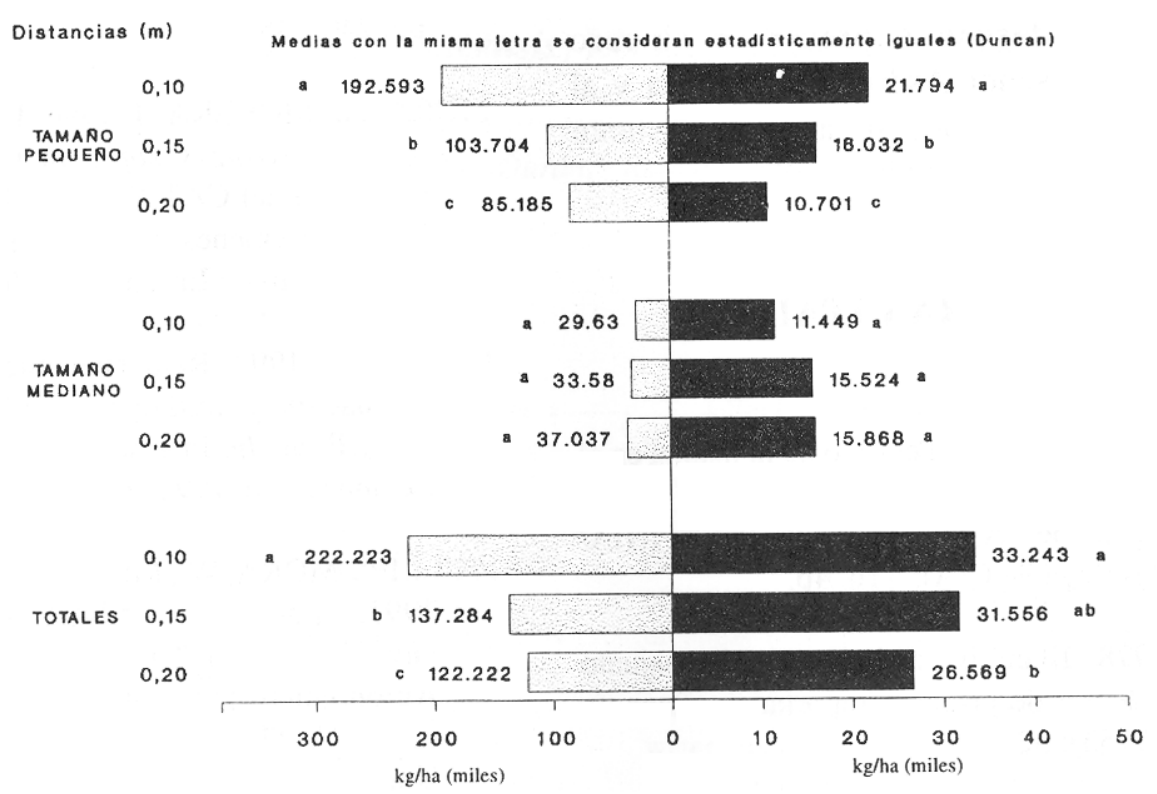

Fig. 3. Promedios de rendimiento por tamaño de raíces y totales para las distancias de siembra evaluadas en Turrialba con tres introducciones de jícama. CATIE. 1993.

crecer y desarrollar más a la distancia mayor (menor densidad) debido a una menor competencia entre plantas.

Al comparar el número de raíces de tamaño pequeño con respecto al tamaño mediano se puede observar que la diferencia es alta en las tres distancias pero no sucede lo mismo en cuánto al peso, donde incluso a $0,20 \mathrm{~m}$ el peso es más alto con las raíces de tamaño mediano.

Para el número de raíces totales es alta la diferencia entre distancias. A $0,10 \mathrm{~m}$ el total de raíces supera por 84939 raíces a la distancia de $0,15 \mathrm{~m}$ y por 100000 raíces a la distancia de $0,20 \mathrm{~m}$. Los pesos totales no muestran estas diferencias tan marcadas entre distancias aunque si es estadísticamente diferente entre $0,10 \mathrm{~m}$ y $0,20 \mathrm{~m}$ (Figura 3). Los resultados para los totales muestran una clara influencia del tamaño pequeño donde las diferencias a favor de este tamaño con respecto al mediano son amplias tal como se mencionó anteriormente.
La diferencia en el número de raíces totales está en función al número de plantas por hectárea que resultan de cada densidad de siembra. Es de esperar que a mayor densidad de plantas más raíces se producirán considerando que cada planta de jícama por lo general produce una sola raíz. Se ha observado que algunas pocas plantas presentan dos o más raíces.

Obtener altos rendimientos con bajas distancias de siembra (alta densidad) no significa que éstas son la mejor alternativa pues estos rendimientos dependen de un alto número de raíces de tamaño pequeño y no comerciales como en el caso de la distancia de $0,10 \mathrm{~m}$ con alta cantidad de raíces menores de 200 g. Aunque no está claramente definido el tamaño óptimo o comercial de las raíces de jícama Paull citado por Phillips-Mora et al (1993) considera, entre otras características, que cada raíz debe pesar más de $300 \mathrm{~g}$ pero menos de $1,1 \mathrm{~kg}$. 


\section{AGRADECIMIENTO}

Los autores desean expresar su sincero agradecimiento al señor Asdrúbal Chavarría y al Ing. Patricia Quesada por su apoyo constante durante la ejecución del estudio.

\section{LITERATURA CITADA}

ARGUELLES, AD. 1979. El cultivo de lajícama en el estado de Guanajuato. Guanajuato, México, Centro de Investigaciones Agrícolas de El Bajío, INIA/SART. Desplegable CIAB 116. 4p.

DIAZ A, A 1978. El cultivo de lajícama: Guanajuato estado productor de primera importancia. Panagfa (México) 6 (53): 32 .

HEREDIA Z., A 1985. Guía para producir jícama en al Bajío. Folleto para productores no. 15. Secretaría de Agricultura y Recursos Hidráulicos (SARH). Instituto Nacional de Investigaciones Agrícolas. Centro de Investigaciones Agrícolas del Bajío. Guanajuato, México. IIp.
KAY, D .E. 1973. TPI crop and product digest. No. 2 Toot Crops. Tropical Products Institute, London, England. pp. 240-245.

MORA, A; MORERA, J. 1994. Evaluación de la jícama (Pachyrhizus spp.) en CATIE. In: Semana Científica del CATIE. (la., 1993. Turrialba, Costa Rica). Resúmenes. Centro Agronómico Tropical de Investigación y Enseñanza. Vo!. 1: 73-74.

MORERA, J. 1992. Rejuvenecimiento, caracterización, evaluación y utilización de jícama en CATIE, Costa Rica. In: International Symposium on Tuber Legumes. (lo. 1992. Guadeloupe). Proceeding.

PHILLIPS-MORA, W.; MORERA, J.; SORENSEN, M. 1993. Las jícamas silvestres y cultivadas (Pachyrhizus spp.) Turrialba, Costa Rica. Centro Agronómico Tropical de Investigación y Enseñanza (CATIE). Royal Veterinary and Agricultura!. University of Denmark. 85p.

SAHADEVAN, N. 1987. Yam bean (Pachyrhizus erosus). In: Sahadevan, N., ed. Green fingers. Malaysia, Sahádevan. p.208-209. 